\title{
Development of Nationwide Road Quality Map: Remote Sensing Meets Field Sensing
}

\author{
Sadra Karimzadeh ${ }^{1,2,3, *(\mathbb{D})}$ and Masashi Matsuoka ${ }^{3}$ (D) \\ 1 Department of Remote Sensing and GIS, University of Tabriz, Tabriz 5166616471, Iran \\ 2 Institute of Environment, University of Tabriz, Tabriz 5166616471, Iran \\ 3 Department of Architecture and Building Engineering, Tokyo Institute of Technology, 4259-G3-2 Nagatsuta, \\ Midori-ku, Yokohama 226-8502, Japan; matsuoka.m.ab@m.titech.ac.jp \\ * Correspondence: sadra.karimzadeh@gmail.com
}

Citation: Karimzadeh, S.; Matsuoka, M. Development of Nationwide Road Quality Map: Remote Sensing Meets Field Sensing. Sensors 2021, 21, 2251. https://doi.org/10.3390/s21062251

Academic Editor: Francisco Valero

Received: 14 February 2021

Accepted: 22 March 2021

Published: 23 March 2021

Publisher's Note: MDPI stays neutral with regard to jurisdictional claims in published maps and institutional affiliations.

Copyright: (C) 2021 by the authors. Licensee MDPI, Basel, Switzerland. This article is an open access article distributed under the terms and conditions of the Creative Commons Attribution (CC BY) license (https:/ / creativecommons.org/licenses/by/ $4.0 /)$.

\begin{abstract}
In this study, we measured the in situ international roughness index (IRI) for first-degree roads spanning more than $1300 \mathrm{~km}$ in East Azerbaijan Province, Iran, using a quarter car (QC). Since road quality mapping with in situ measurements is a costly and time-consuming task, we also developed new equations for constructing a road quality proxy map (RQPM) using discriminant analysis and multispectral information from high-resolution Sentinel-2 images, which we calibrated using the in situ data on the basis of geographic information system (GIS) data. The developed equations using optimum index factor (OIF) and norm $R$ provide a valuable tool for creating proxy maps and mitigating hazards at the network scale, not only for primary roads but also for secondary roads, and for reducing the costs of road quality monitoring. The overall accuracy and kappa coefficient of the norm R equation for road classification in East Azerbaijan province are $65.0 \%$ and 0.59 , respectively.
\end{abstract}

Keywords: international roughness index; road quality; remote sensing; Sentinel-2

\section{Introduction}

Since ancient times, roads have played an important role in connecting different geographic locations to each other. Currently, in the face of rapid urbanization and industrialization, the role of roads is even more important because they not only allow people to travel from one location to another but also contribute to the economic growth of nations [1]. If the surface evenness, rideability, quality, skid resistance, or other influential factors are below the defined standards, a road can be fixed or require maintenance [2]. Poorly maintained roads can seriously affect economy, fuel consumption, and mobility, while further increasing driving costs, air pollution, and maintenance/operating costs for both roads and vehicles. From this economic perspective, road quality mapping is an important task for enabling safe, smooth, and efficient travel. Roads with better quality also enable farmers to work in more distant fields and distribute their products more efficiently.

For the general population, "road quality" denotes the level of roughness of the road, along with other parameters, such as number of lanes, potholes, signage and layout. Several roughness indices have been developed to date. The international roughness index (IRI), introduced in the early 1980s, is one such index that is basically obtained from the measured longitudinal road profile [3-5]. The IRI is a measure of the displacement in the vertical direction per horizontal distance traveled on the road. In other words, the IRI, which is expressed in units of meters/kilometer or inches/mile, represents a roughness quality that affects vehicle performance on the road. Although ground-based methods for measuring the IRI are of most interest, remote sensing methods are emerging as an alternative for acquiring in situ IRI measurements. Remote sensing techniques are capable of measuring various types of information without physical contact (only through electromagnetic waves). Therefore, remote sensing measurements can be recorded using 
various platforms, such as unmanned aerial vehicles (UAVs), airplanes, and satellites, from which many infrastructure changes can be detected and monitored. A few examples of such remote sensing applications to infrastructure monitoring include observing infrastructure damage after natural and man-made disasters, planning long-term road consolidation, and detecting dam instabilities [6-15].

Figure 1a shows the total length (approximately $214,000 \mathrm{~km}$ ) of the road network in Iran, including primary (freeway and highway), secondary (major and local), and rural roads. East Azerbaijan Province, one of the 31 provinces in Iran with a road network extending up to approximately $14,000 \mathrm{~km}$, plays an important role in national road networking because it connects Iran to Armenia and the Republic of Azerbaijan. In this work, we measured the IRI on major roads throughout East Azerbaijan Province of Iran using a smartphone equipped with a motion-sensitive "BumpRecorder" and "Physics Toolbox" applications and a quarter car (QC). The BumpRecorder application did not require any specific calibration, since its accuracy was tested several times before the commercial operation [16]. These measurements were acquired by driving back and forth only between the capital of the province, Tabriz, and the counties shown in yellow in Figure 1b. The road map of East Azerbaijan Province (Figure 1b) is produced from the OpenStreetMap (OSM) database. Using in situ data as training samples, combined with remote sensing data (featuring a broader coverage of the desired region), incomplete information about different objects or features can be completed. Accordingly, we regressed independent variables obtained from a Sentinel-2 dataset on the dependent variables (field data) to analyze the road network of the whole country.
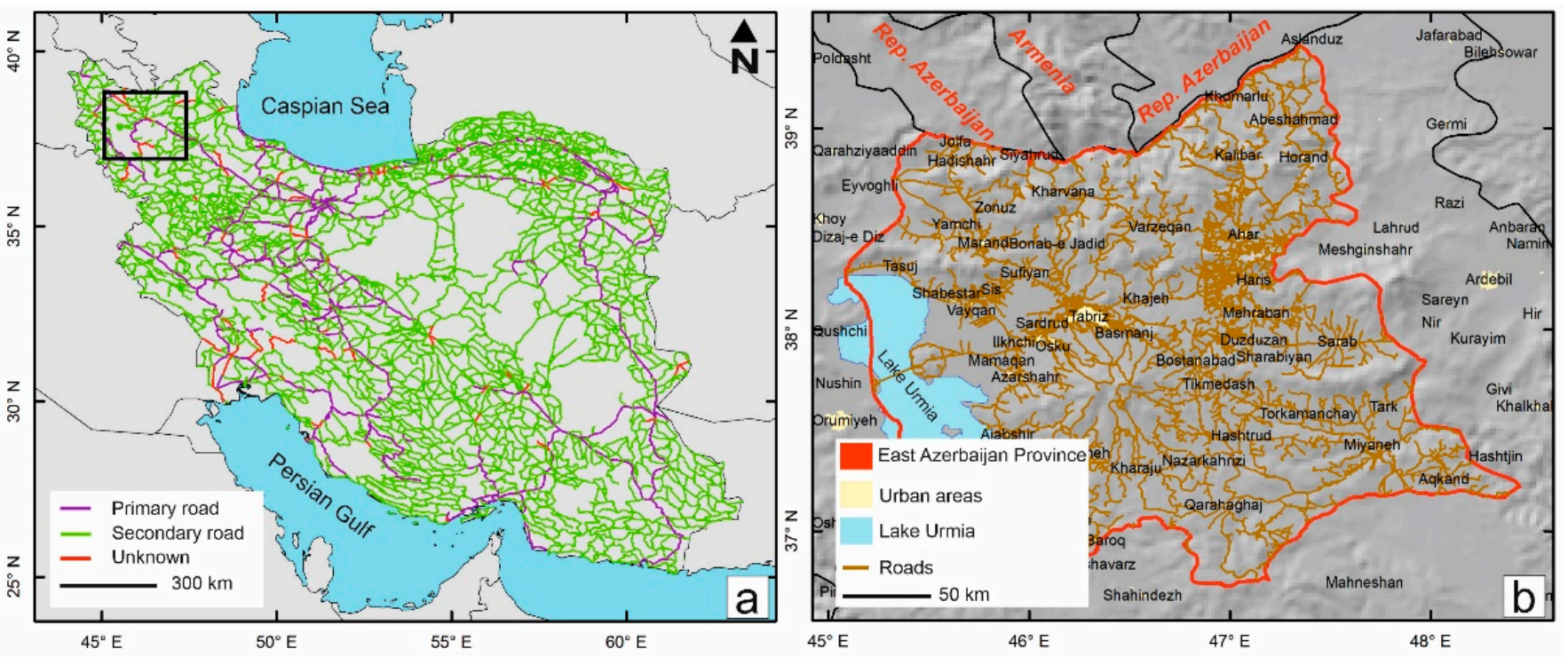

Figure 1. (a) Road network map of Iran. The black box denotes the study area. (b) Road network of East Azerbaijan Province, Iran, the study area.

\section{Data and Methodology}

\subsection{In Situ Measurements}

Engineers working in the transportation sector typically use a "one-wheel" vehicle to acquire traditional field measurements of road quality. These vehicles are suitable for precise IRI measurements because the roughness measurements are not affected by the suspension system $[16,17]$. In contrast, the QC method was not originally popular among engineers because the QC has a suspension system. Therefore, due to this suspension system, the recorded acceleration might be affected by the car type and driving speed. The roughness measurements recorded with a QC are also not typically stable, and it is necessary to perform a driving calibration before taking measurements. However, the extensive use of smartphones has marked a paradigm shift for road quality mapping. 
In this study, we used the BumpRecorder application developed by BumpRecorder Co., Ltd., to measure the acceleration and longitudinal profile of the road and refine the results using vibration frequency analysis. The use of this application therefore eliminates the need for a driving calibration step. Although the type of car does not seriously affect the measurements, we used only one car for the entire project. As shown in Figure 2a, we mounted a smartphone (Huawei Y7) equipped with an accelerometer and a global positioning system (GPS) sensor on the dashboard tray of a long chassis car (CHANGAN-CS35) to acquire IRI measurements on the roads (e.g., highways and major roads) throughout East Azerbaijan Province.
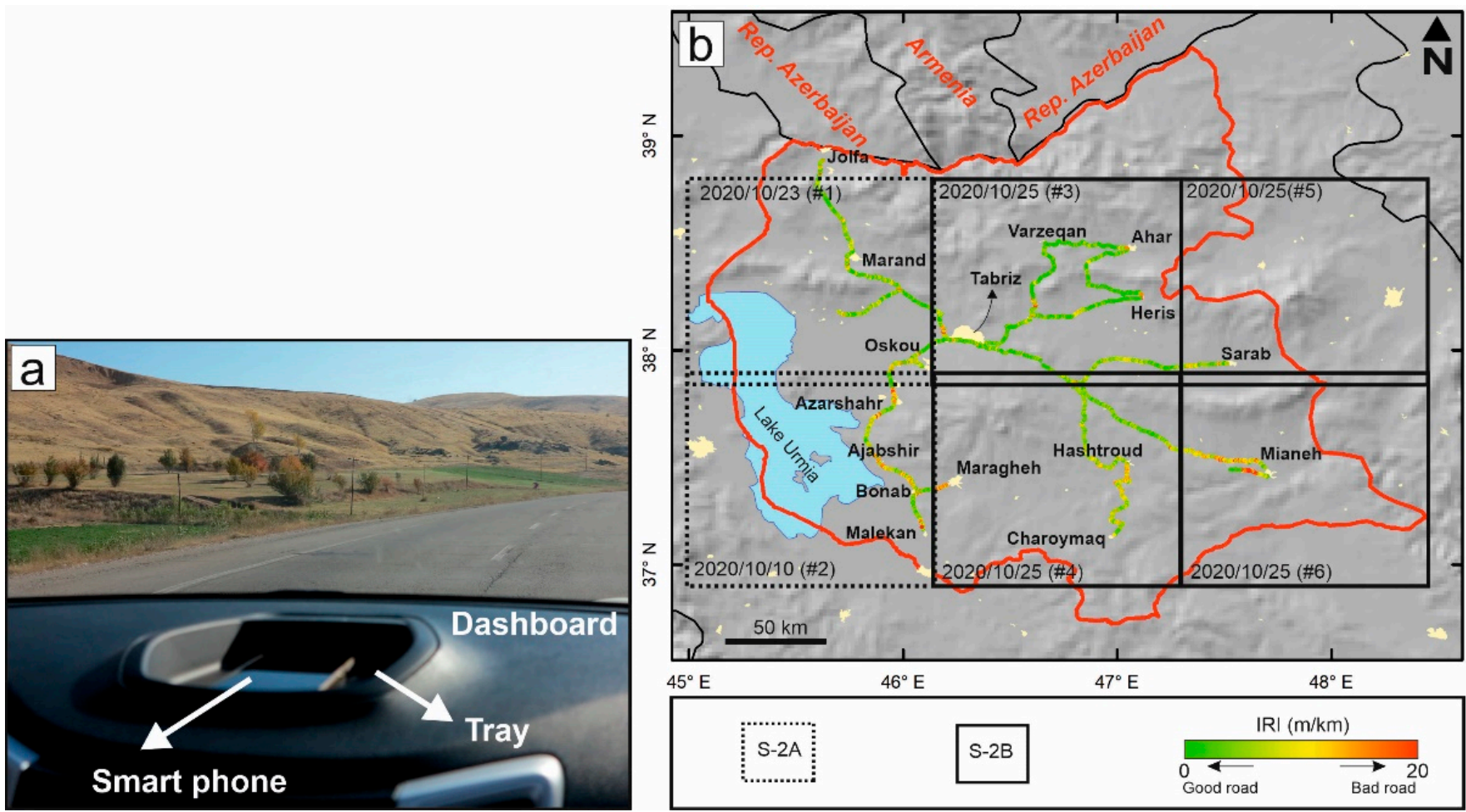

Figure 2. (a) Setup for conducting international roughness index (IRI) measurements in East Azerbaijan Province using a quarter car (QC) and a smartphone. (b) The resulting back-and-forth in situ IRI measurements in East Azerbaijan Province of Iran from Tabriz along the primary roads leading to the other counties. Black boxes show the areas covered by Sentinel-2 images of the province. Dashed boxes indicate the images acquired from Sentinel-2A, and solid black boxes indicate the images acquired from Sentinel-2B.

Unfortunately, even with a QC, the measurement of major roads is time-consuming because there are often several lanes and driving on the same road several times to record the quality of each lane requires additional labor and time. To reduce the time required to perform these ground-based in situ measurements, we drove on only one lane on each road, with priority given to the lane with the worst road quality. Due to the cold winters and the mountainous topography of the study area, the measurements were gathered from 13 October 2020 to 30 October 2020 to avoid dangerous driving conditions. We took measurements on major roads spanning more than $1300 \mathrm{~km}$ throughout East Azerbaijan Province, Iran, with an average speed of $80 \mathrm{~km} / \mathrm{h}$ and a sampling frequency of $200 \mathrm{~Hz}$. According to Karimzadeh and Matsuoka (2020), the accuracy of the QC method is acceptable for major roads, but on urban streets, tall buildings and traffic jams may reduce the quality of simultaneous location and acceleration measurements [4]. Figure $2 \mathrm{~b}$ shows the IRI field measurements every $200 \mathrm{~m}$, in which green, yellow-orange, and red colors represent smooth roads, intermediately rough roads, and the roughest roads, respectively. 
According to Sayers and Gillespie (1986), roads with IRI values between 1 and $4 \mathrm{~m} / \mathrm{km}$ denote new pavement [5]. Figure $3 a, b$ show that the IRI was recorded at 7618 points. The minimum and maximum in situ IRI values are $0.9 \mathrm{~m} / \mathrm{km}$ and $18.4 \mathrm{~m} / \mathrm{km}$, respectively. The mean and median of the measurements are $3.13 \mathrm{~m} / \mathrm{km}$ and $2.8 \mathrm{~m} / \mathrm{km}$, respectively, which implies that the major roads across the province have good overall quality. The roads studied are well maintained except for several routes. Figure $3 \mathrm{c}$ illustrates the distribution of IRI measurements in nonsymmetrical regions, and the first and third quartiles are $2.3 \mathrm{~m} / \mathrm{km}$ and $3 / 6 \mathrm{~m} / \mathrm{km}$, respectively. The probability-probability plot (P-P) shown in Figure $3 \mathrm{~d}$ can be used to test the fitness of theoretical and empirical distributions and to depict the skewness of the IRI distribution, which is not quite normal here (positive skew).
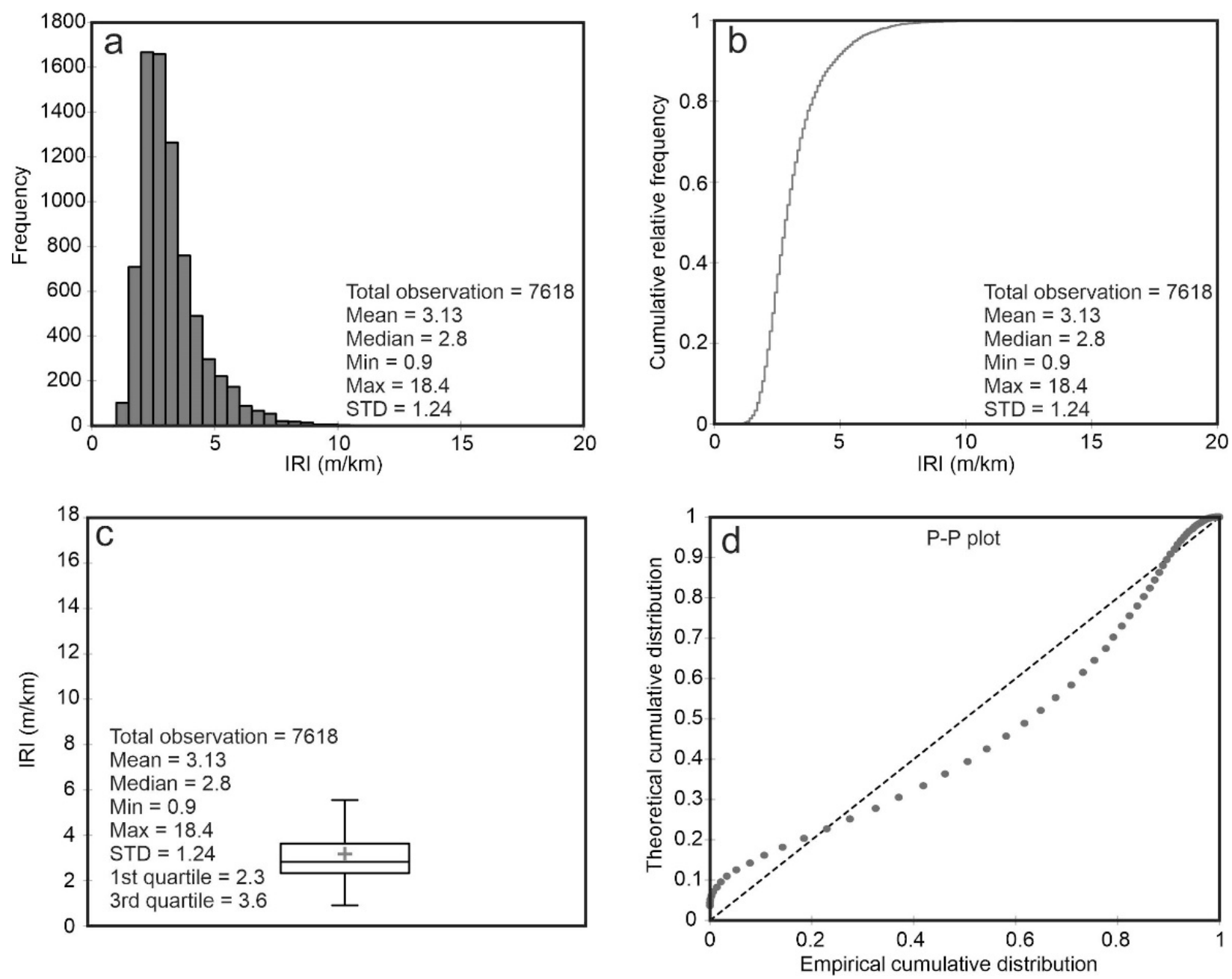

Figure 3. (a) Histogram of IRI measurements for the study area. (b) Empirical cumulative distribution of IRI measurements. (c) Boxplot of IRI measurements. The "+" sign indicates the mean IRI value. (d) Probability-probability (P-P) plot of the empirical cumulative distribution versus the theoretical cumulative distribution.

\subsection{Remote Sensing Data}

Remote sensing optical data obtained from UAVs or airborne/spaceborne platforms are mainly used for the extraction of roads, as described in several previous studies [18-22] Meanwhile, synthetic aperture radar (SAR) data, especially high-resolution X-band SAR datasets, focus on the quality of the road. In previous studies, such high-resolution X-band SAR datasets were compared with IRI data in the U.S. and Iran, where bad-quality roads appear brighter in the SAR images than good-quality roads because rough surfaces have stronger backscattering properties [3,4]. However, despite the rapid growth of remote sensing, many transportation engineers have insufficient experience to practically apply 
remotely sensed data for their demands. On the other hand, remote sensing experts are not familiar with the engineering demands within the transportation sector. The interpretation of SAR data is more complex than that of optical data due to the sidelooking nature of the former and its inherent limitations, such as foreshortening. Here, we focus on high-resolution optical data from Sentinel-2A and B within the study area and conduct novel research to develop equations that explain the roughness of primary and secondary roads. As mentioned above, we measured over $1300 \mathrm{~km}$ of roads in East Azerbaijan Province of Iran. To cover all the measured areas, we obtained 6 Sentinel-2 images $(100 \mathrm{~km} \times 100 \mathrm{~km})$ with the closest available timestamps to each day on which the in situ measurements were obtained, from 10 October 2020 to 25 October 2020. The Sentinel2 imagery has 12-bit radiometric resolution, $10 \mathrm{~m}$ spatial resolution, and 13-band spectral resolution. The bottom-of-atmosphere (BOA) reflectance values in the images from the MultiSpectral Instrument (MSI) were extracted from the 1C products of Sentinel-2, which are not atmospherically corrected. The Sentinel-2 mission has two polar-orbiting satellitesnamely, Sentinel-2A and Sentinel-2B. As shown in Figure 2b and Table 1, two Sentinel-2 images (acquired on 10 and 23 October 2020) were obtained from Sentinel-2A, while the remaining images (25 October 2020) were obtained from Sentinel-2B. The mosaicked image covers approximately $90,000 \mathrm{~km}^{2}$ of the study area. Except for a gap between images \#1 and $\# 3$, the regions containing the IRI measurements are well covered in the mosaicked image. Once the radiometric calibration of the mosaicked image is performed, the optimum bands that contain the highest variance (i.e., information) can be selected using the optimum index factor (OIF).

Table 1. Detailed information about the Sentinel-2 images used for this study. The MSI2A instrument provides bottom-of-atmosphere (BOA) reflectance values for the Sentinel-2 images.

\begin{tabular}{ccccc}
\hline ID (\#) & Sensor & Product Type & Date $(\mathbf{y y y y} / \mathbf{m m} / \mathbf{d d})$ & Area $\left.\mathbf{( k m}^{\mathbf{2}}\right)$ \\
\hline 1 & Sentinel-2A & MSI2A & $2020 / 10 / 10$ & 10,000 \\
2 & Sentinel-2A & MSI2A & $2020 / 10 / 23$ & 10,000 \\
3 & Sentinel-2B & MSI2A & $2020 / 10 / 25$ & 10,000 \\
4 & Sentinel-2B & MSI2A & $2020 / 10 / 25$ & 10,000 \\
5 & Sentinel-2B & MSI2A & $2020 / 10 / 25$ & 10,000 \\
6 & Sentinel-2B & MSI2A & $2020 / 10 / 25$ & 10,000 \\
\hline
\end{tabular}

\subsection{Framework}

The framework shown in Figure 4 shows the details of each step-namely, data calibration, discriminant analysis, and accuracy assessment.

\subsubsection{Optimum Index Factor}

The OIF is a measurement that is calculated for selecting the most ideal bands to be used for color composites or for preserving the maximum amount of information (highest standard deviation) in an image with minimal duplication [23-25]. The OIF calculation method starts with the creation of a map list that contains 13 Sentinel-2 bands. Once this list of multispectral bands is available, the variance-covariance matrix (correlation matrix) of the bands can be calculated according to the map list. To create any OIF, the map list should contain at least 3 raster images that are all georeferenced in the same domain. A simple OIF calculation method is as follows:

$$
\mathrm{OIF}=\frac{\sum_{k=1}^{3} s_{k}}{\sum_{j=1}^{3} A b s\left(r_{j}\right)}
$$

where $s_{k}$ is the standard deviation for the corresponding band $(k)$ and $j$ is the absolute value of the correlation coefficient between two of the three bands in the calculation. 


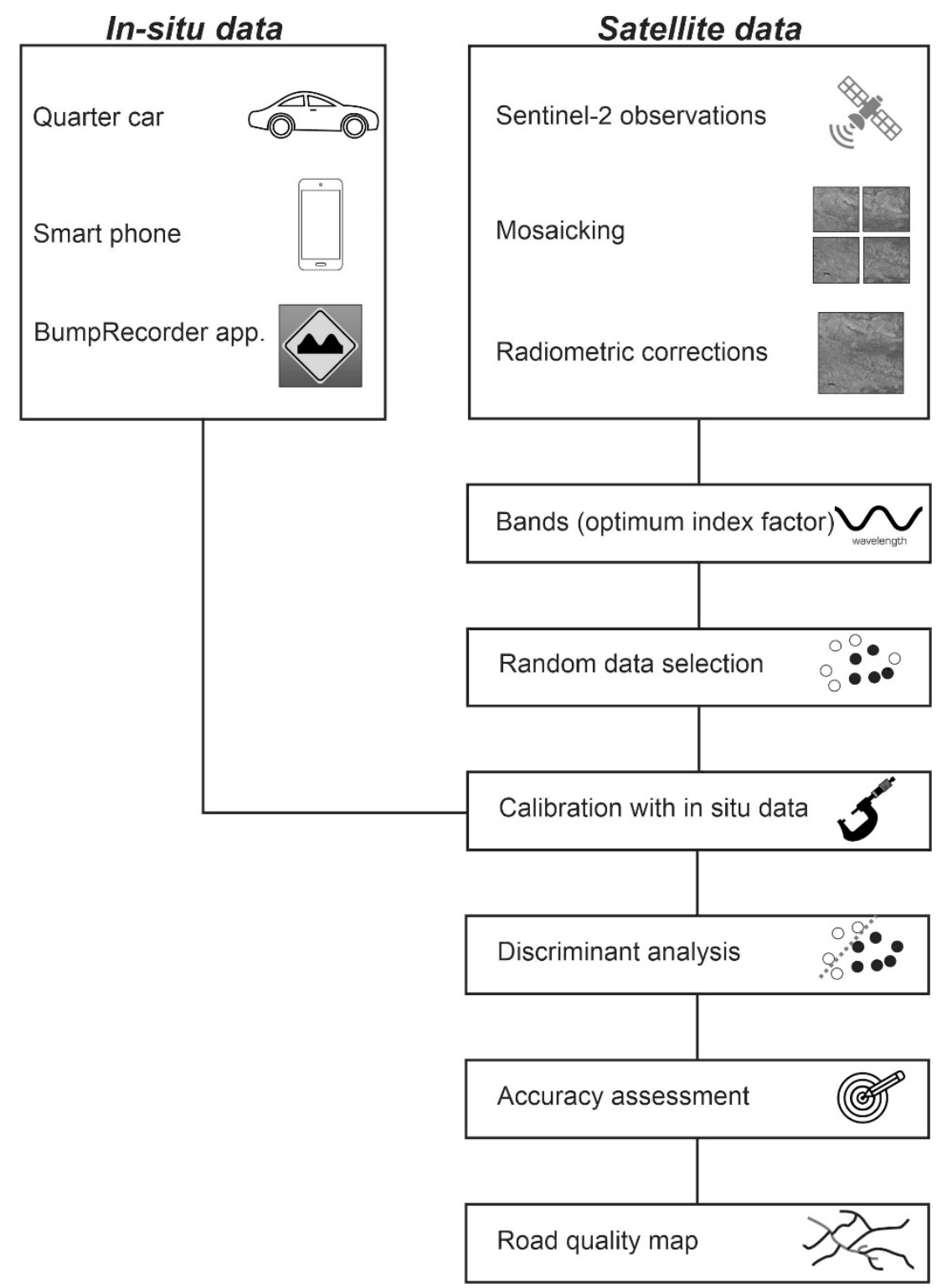

Figure 4. Workflow of road quality mapping based on in situ and satellite datasets.

\subsubsection{Discriminant Analysis}

In this study, the discriminant analysis was structured based on independent variables (i.e., spectral bands such as $b_{2}, b_{3}, b_{4}$, and $b_{8}$ ) and a binary map (the dependent variable). Note that for the binary map of the study area, " 0 " was assigned to good roads and " 1 " was assigned to bad roads. The binary map was produced from in situ IRI measurements conducted using a QC. Although these IRI measurements varied in the range between $0.9 \mathrm{~m} / \mathrm{km}$ and $18.4 \mathrm{~m} / \mathrm{km}$, a simple assumption (e.g., good and bad roads) helped us to categorize the values into two qualitative groups: 0 for good roads and 1 for bad roads. Thus, a qualitative rather than a quantitative discriminant analysis was chosen to predict new scores based on the independent variables and the description of the binary map [26-29]. Here, discriminant analysis is a type of linear regression that seeks to find the relationship among several classes of objects. We regressed the independent variables for both the OIF and norm $R$ results on the dependent variables and assigned a specific value for each sample point in the ground truth database. Table 2 shows the details of the linear 
regression for the independent and dependent variables. The discriminant score $(Z)$ for the OIF and norm $\mathrm{R}$ can be defined as follows:

$$
\begin{gathered}
\mathrm{Z}_{\mathrm{OIF} \_}=a_{1}+\left(a_{2} \times b_{i 2}\right)+\left(a_{3} \times b_{i 3}\right)+\left(a_{4} \times b_{i 4}\right)+\left(a_{5} \times b_{i 8}\right) \\
Z_{R_{-} i}=a_{1}+a_{2} \times N_{i}
\end{gathered}
$$

Table 2. Statistics for the linear regression analysis of the optimum index factor (OIF) and norm $R$ dependent and independent variables.

\begin{tabular}{cccc}
\hline \multicolumn{1}{c}{ OIF $\mathbf{( 4 \mathbf { 1 } \times \mathbf { 4 1 } )}$} & \multicolumn{2}{c}{ Norm R $\mathbf{( 2 1 \times \mathbf { 2 1 } )}$} \\
\hline Number of points & 7313 & Number of points & 7313 \\
R-squared & 0.026 & R-squared & 0.0007 \\
Multiple R & 0.163 & Multiple R & 0.0275 \\
Standard error & 0.4 & Standard error & 0.405 \\
$a_{1}$ (intercept) & 0.317 & $a_{1}$ (intercept) & 0.342 \\
$a_{2}$ & 0.00138 & $a_{2}$ & -0.425 \\
$a_{3}$ & -0.00149 & $a_{3}$ & - \\
$a_{4}$ & 0.0002 & $a_{4}$ & - \\
$a_{5}$ & 0.000087 & $a_{5}$ & - \\
Cutoff score & 0.211 & Cutoff score & 0.207 \\
\hline
\end{tabular}

Here, $i$ represents the number of points on the IRI map. Because of the image gap (Figure $2 b$ ), the total number of points is reduced from 7618 to $7313 . a_{1}$ is the intercept value, and $a_{2}, a_{3}, a_{4}$, and $a_{5}$ are coefficients of the regression analysis for the blue, green, red, and near-infrared bands, respectively. $Z_{O I F_{-} i}$ and $Z_{R_{-} i}$ are the probability values for the OIF and norm $R$, respectively, which can be interpreted as the probabilities of being a "good" or a "bad" road. To define whether the calculated values are in the good or bad groups, we defined a cutoff value for the calculated discriminant scores as follows:

$$
C=\frac{\left(n_{0} \times \bar{z}_{0}\right)+\left(n_{1} \times \bar{z}_{1}\right)}{\left(n_{0}+n_{1}\right)}
$$

where $C$ is the cutoff value, $n_{0}$ and $n_{1}$ are the numbers of good and bad roads, respectively, and $\bar{z}_{0}$ and $\bar{z}_{1}$ are the average values of the discriminant scores for good and bad roads, respectively. As shown in Table 2, the cutoff values of the OIF and norm R classifications are 0.211 and 0.207 , respectively. This means that the roads with a lower value than the cutoff were sorted into group " 0 " and the roads with a higher value than the cutoff were sorted into group " 1 ". In other words, if a point from the database has a $Z$ value smaller than the calculated $C$, the algorithm will categorize it as a "good" point (road), whereas if one point from the database has a $Z$ value larger than the calculated $C$, the algorithm will categorize it as a "bad" point.

\section{Results}

Generally, we found an acceptable fit between the spectral information within the Sentinel-2 images and the in situ measurements, but the road quality map generated from the latter (graded colors in Figure $2 b$ ) was not helpful for discriminant analysis. To produce meaningful results for facile interpretation, the IRI measurements should be presented in a simpler format than the format traditionally used for these road quality maps. Due to the level of noise and the standard deviation of the measurements, as shown in Figure 5, all of the IRI measurements were categorized into two main classes: "good" and "bad" roads. Since road maintenance guides do not clearly define the metrics for classifying good and bad roads, we used the Jenks natural break optimization thresholding approach. This method is a data clustering technique for determining the most ideal arrangement of the IRI values into good and bad classes. Notably, Jenks optimization operates on a univariate classification, which maximizes the differences between the good and bad classes. Simply 
put, this method attempts to reduce the intraclass variance while maximizing the interclass variance. This goal is logical for our work because the IRI measurements can be divided into two categories where the difference in the IRI is relatively large. We adopted the following threshold for the classification of every measurement point:

$$
\text { IRI }_{\text {threshold }}=\left\{\begin{array}{c}
\text { Good road : IRI }<3.8 \mathrm{~m} / \mathrm{km} \\
\text { Bad road : IRI } \geq 3.8 \mathrm{~m} / \mathrm{km}
\end{array}\right.
$$
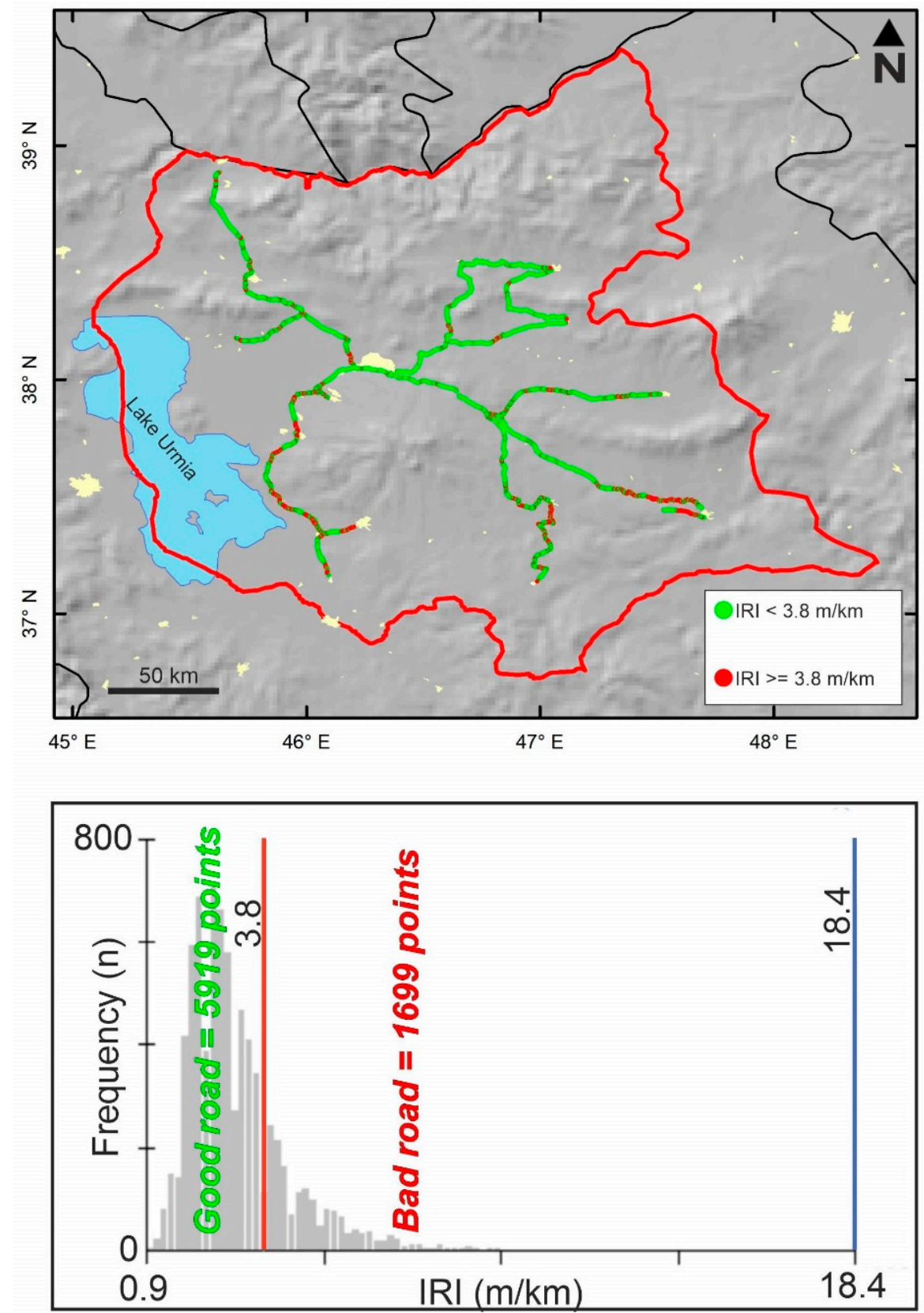

Figure 5. Binary road quality map based on a Jenks classification for IRI measurements.

As we divided the IRI measurements into two groups (Figure 5), we also created a binary map using discriminant analysis which is an essential step for the analysis. Sentinel2 images are composed of 13 bands at resolutions of $10 \mathrm{~m}, 20 \mathrm{~m}$ and $60 \mathrm{~m}$. Based on the OIF, we selected useful bands that maintained the highest variance and ignored redundant information. These OIF results showed that the combination of bands 2, 3, 4 and 8 could 
provide the most information. Thus, discriminant analysis in different window sizes from $3 \times 3$ to $57 \times 57$ was carried out for these bands. The results show that among the 7618 IRI points, 5919 points denote good roads, and 1699 points signify bad roads (Figure 5). To apply the discriminant machine learning approach to the binary classification map, we employed a statistical measure - namely, the OIF-which combines only the useful multispectral bands in terms of the explained variance and standard deviation $[23,24]$. The OIF was utilized to reduce the complexity of the multispectral Sentinel-2 dataset and improve the discrimination accuracy for good and bad roads. The combination of the three bands with the largest OIF values as a red, green, blue (RGB) color composite yielded the most useful information while minimizing the amount of duplicated information. Here, we found that bands 2, 3, 4 and 8 had the most information; among them, the combination of bands 3,4 and 8 yielded the largest OIF equal to 43.656 . The second-largest OIF was produced by the combination of bands 2,4 and $8(\mathrm{OIF}=39.970)$. The discriminant analysis was conducted in different window sizes. Since the bidirectional reflectance distribution function and mix of pixels (MIXEL) for neighboring features (e.g., road and vegetation) can increase the amount of false spectral information during discriminant analysis, we applied different window sizes to maintain the real reflectance of the road surface [30,31]. The first model was based on the original data $(1 \times 1)$, and the other window sizes were $3 \times 3$, $5 \times 5,7 \times 7,9 \times 9,13 \times 13,17 \times 17,21 \times 21,25 \times 25,29 \times 29,33 \times 33,37 \times 37,41 \times 41$, $47 \times 47$, and $57 \times 57$. As shown in Figure 6, for bands 2, 3, 4 and 8, the most ideal window size for road classification was $41 \times 41$, and the corresponding discriminant equation is as follows:

$$
Z_{i}=0.317+0.00138 b_{2}-0.00149 b_{3}+0.0002 b_{4}+0.000087 b_{8}
$$

where $b_{2}, b_{3}, b_{4}$, and $b_{8}$ are Sentinel-2 bands $2,3,4$ and 8 , respectively. The overall accuracy $(\mathrm{OA})$ of the abovementioned technique for a $41 \times 41$ window is $61.0 \%$ (kappa $\sim 0.51$ ), but the classification of bad roads is still poor (accuracy of $\sim 19 \%$ ). Figure $7 \mathrm{a}$ indicates that the roads were classified according to a normal distribution. Since the total number of points (7313) was less than the number of in situ points (due to the gap in the mosaicked Sentinel-2 image), 7162 points were identified as good roads (green), while 151 points were identified as bad roads (red). The cutoff point was 0.211: values beyond this threshold were grouped into the bad road category. Details of the categorization based on this cutoff value and the corresponding accuracies are given in Table 3.

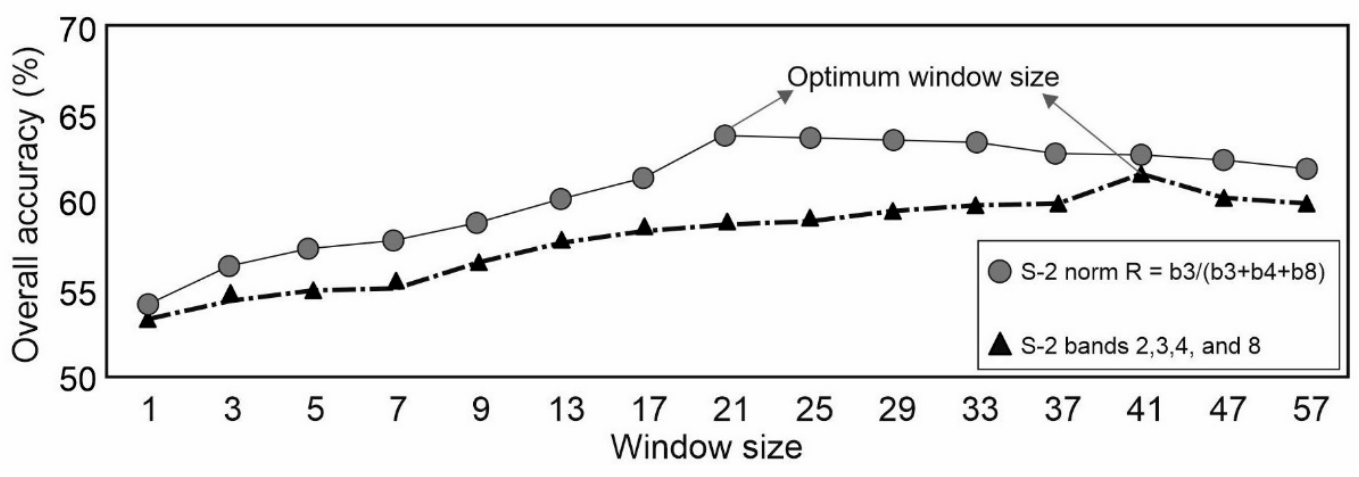

Figure 6. Overall accuracy of the OIF and norm R results using different window sizes. 

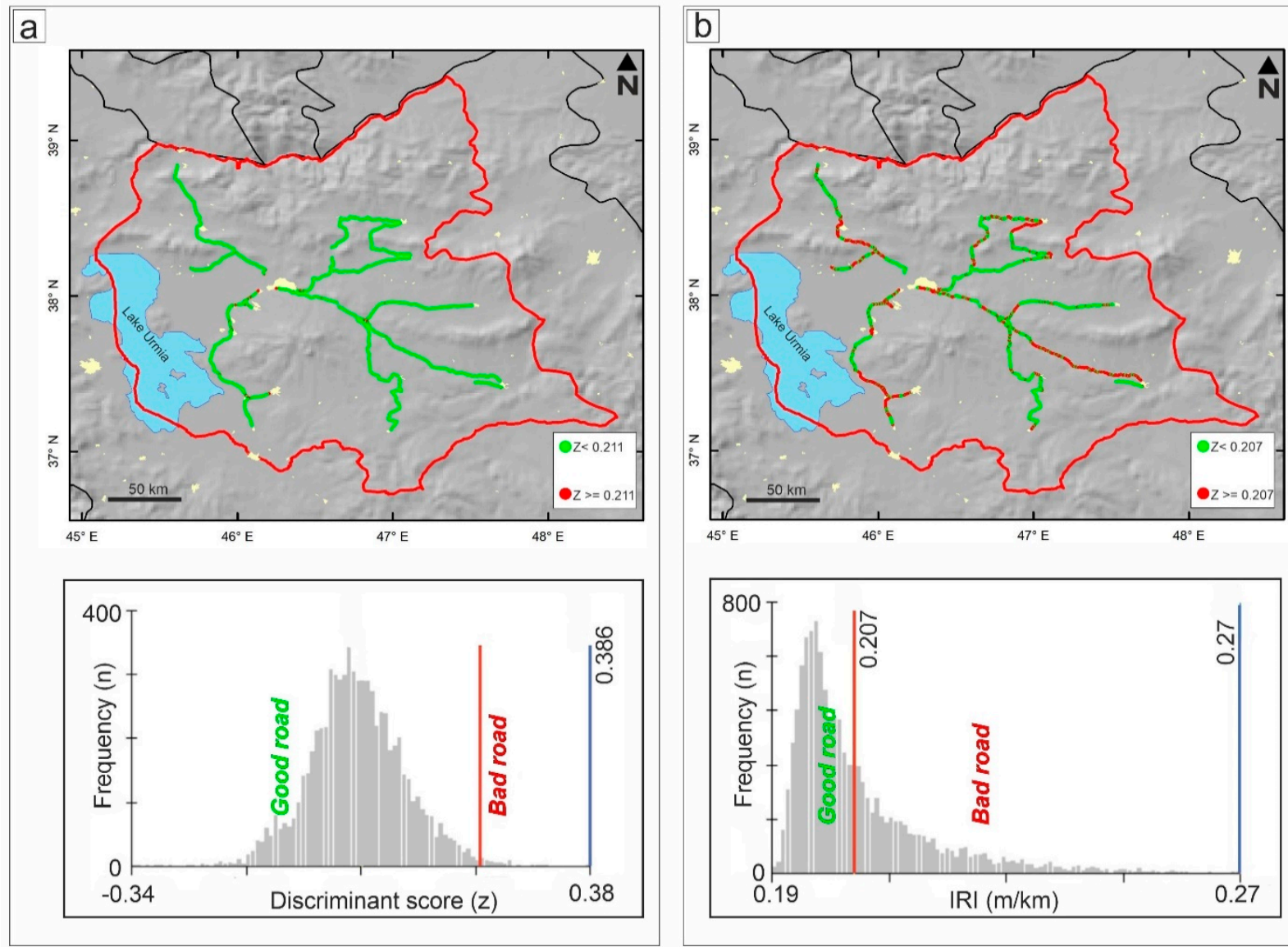

Figure 7. (a) Binary road quality proxy map (RQPM) deduced from the OIF and discriminant analysis. (b) Binary RQPM deduced from the norm $\mathrm{R}$ and discriminant analysis.

Table 3. Road classification accuracy based on the discriminant scores $\left(Z_{i}\right.$ and $\left.Z_{R}\right)$, optimum index factor (OIF) and norm $\mathrm{R}$.

\begin{tabular}{cccc}
\hline \multicolumn{2}{c}{ Sentinel-2 Classification (OIF) } & \multicolumn{2}{c}{ Sentinel-2 Classification (Norm R) } \\
\hline$Z_{i}>0.211$ & 7162 & $Z_{R}>0.207$ & 4641 \\
$Z_{i} \leq 0.211$ & 151 & $Z_{R} \leq 0.207$ & 2672 \\
Misclassified roads & 2852 & Misclassified roads & 2560 \\
Total correct roads & 4461 & Total correct roads & 4753 \\
Total accuracy & $61 \%$ & Total accuracy & $65 \%$ \\
\hline
\end{tabular}

As an alternative, we applied another index (norm R) introduced by https: / www. indexdatabase.de (access date 15 December 2020). Here, the norm R index was selected based on empirical tests of replacement of the bands. This index was suitable for our purposes since it is simpler than the combination of OIF bands and, like the OIF, it contains the maximum amount of information because the red, green, and NIR (near-infrared) bands all contribute to this index. The norm $\mathrm{R}$ is defined as follows:

$$
N=\frac{b_{3}}{b_{8}+b_{4}+b_{3}}
$$

where $N$ is the norm $\mathrm{R}$ and $b_{3}, b_{4}$ and $b_{8}$ are the green, red and near-infrared bands of Sentinel-2, respectively. Figure 6 shows the OA for different window sizes. Equation (8) is the discriminant equation for the optimum window size $(21 \times 21)$ produced from the norm $\mathrm{R}$ index:

$$
Z_{R}=0.342-0.425 \times N
$$


Figure $7 \mathrm{~b}$ indicates that, using this index, the roads are classified with a skewed distribution. Based on the estimated cutoff point (0.207), among the 7313 points, 4641 points were identified as good roads, while 2672 points were identified as bad roads. The OA for linear discriminant analysis on a window size of $21 \times 21$ was approximately $65 \%$ (kappa $\sim 0.59$ ), and the classification accuracy of bad roads was improved to $40 \%$. The details of this categorization based on the corresponding cutoff value and its accuracies are given in Table 3. Equations (6) and (8) permit the calculation of road quality proxy maps (RQPMs) for large-scale regions on the basis of the existing raster pixel values and regressed dataset. Figure 8 shows the nationwide RQPM of the territory of Iran produced from Equation (8) and the mosaic of 23 Sentinel-2 images in the Google Earth Engine (GEE) for bands 2, 3, 4 and 8 during October 2020.

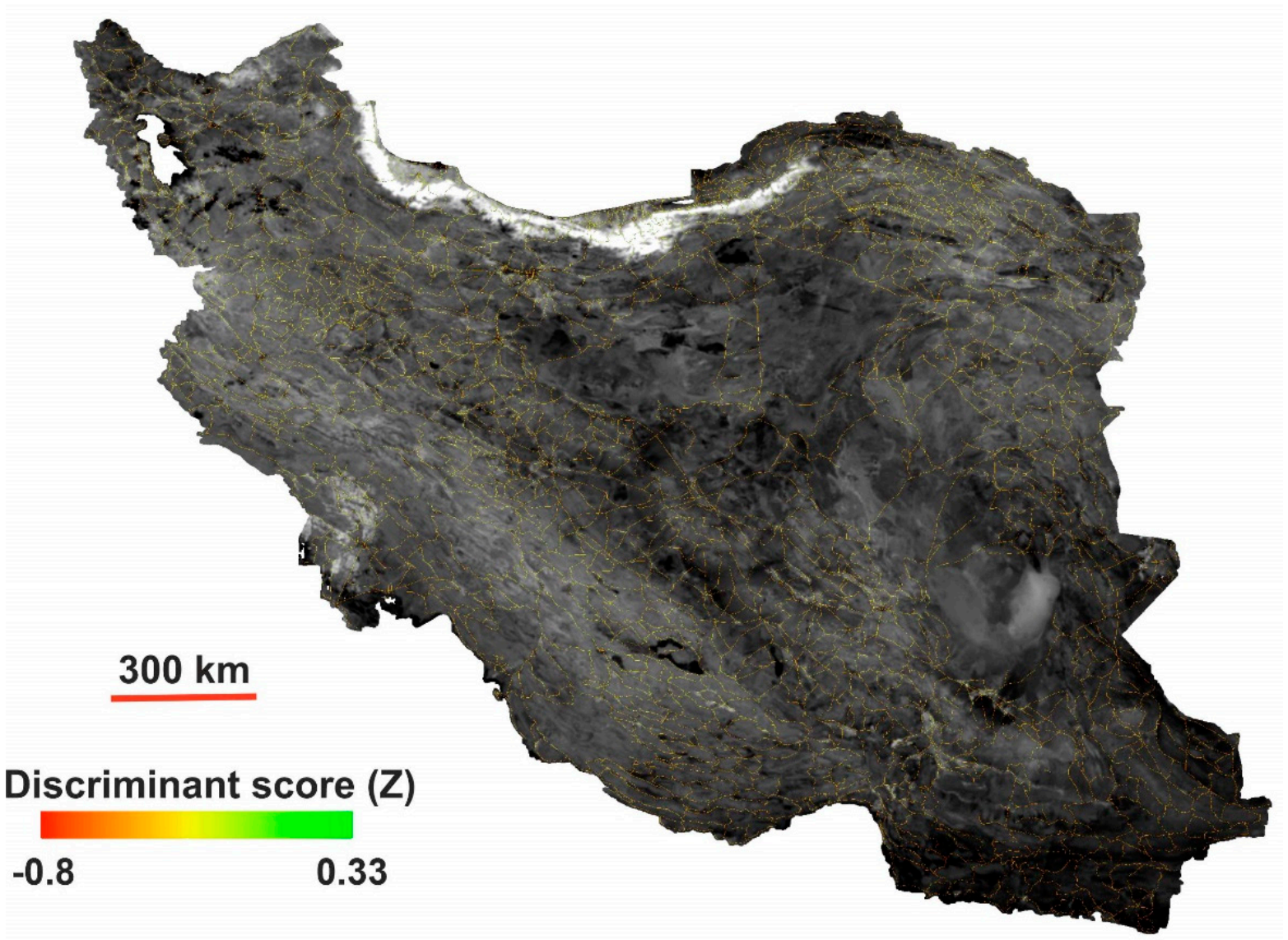

Figure 8. Nationwide RQPM deduced from the discriminant model of norm R.

\section{Discussion}

Discovering local damages or local road unevenness using Sentinel-2 dataset $(10 \mathrm{~m}$ spatial resolution) is challenging. Additionally, in dense urban areas surrounded by tall buildings and trees, IRI measurements could be biased (due to weak GPS signals, traffic jams, etc.). Thus, the field data gathered from highways and first-degree roads (every $200 \mathrm{~m}$ ) together with Sentinel-2 spectral information can be used for road quality mapping over large-scale areas. Further investigations using very high resolution (VHR) images from commercial satellite images (e.g., WorldView-2, Spot, etc.) might help to discover different aspects of road quality mapping from space. The developed discriminant models (for OIF and norm R) in Equations (6) and (8) permit the calculation of RQPMs over large-scale areas based on the existing correlation between road roughness and raster pixel values, thereby reducing the hazard mitigation costs and time-consuming task of in situ road quality mapping. Despite its advantages, such as its compatibility with multiple dependent variables, reduced error rate, and simpler interpretation between grouped 
values, there are still some disadvantages to discriminant analysis. One such disadvantage is that discriminant analysis assumes the relationship between variables is linear although the relationship is not exclusively linear, especially for the backscattering coefficient values extracted from X-band SAR images. In particular, the relationship between the IRI and backscattering coefficient is not linear for whole roads (e.g., interstate and secondary roads) because the IRI is measured indirectly, and the relationship between the IRI and pavement roughness is somewhat complicated [3,32]. As mentioned before, the classification accuracy for bad roads is low, which is probably related to the inherent disadvantage of discriminant analysis being sensitive to outliers. Hence, an unbalanced number of points in the good and bad categories could have caused the poor segregation results. In the future, it will be important to create more robust models, increase the number of samples in difficult areas (e.g., with bad roads), and apply post-processing techniques such as generative learning or new machine learning algorithms compatible with any type of dataset.

It must be noted that tall or dense vegetation can negatively affect the results of road quality mapping. Since the study area has semi-arid climate, the vegetation was not a challenging problem. But to reduce the effects of vegetation, the mosaicked Sentinel-2 map was also masked using $10 \mathrm{~m}$ buffering and OSM. Other techniques such as principal component analysis (PCA) might be helpful to discover different aspects of road mapping, but the in situ IRI is a physical parameter that cannot fully be explained by pixel values. In addition, for a nationwide road quality analysis using GEE, calculation of PCA will be more difficult due to limited cloud storage.

\section{Conclusions}

We conclude that while the transportation sector is growing due to many reasons, such as socioeconomic demands, remote sensing application techniques should not only be focused on routine change detection issues but also on monitoring the quality of the features in detail. This study proposed a qualitative remote sensing approach for the segregation of good and bad roads based on IRI measurements and spectral information. We analyzed 4 bands of 6 multispectral Sentinel-2 images over East Azerbaijan Province in Iran and developed linear models to describe the correlation between the spectral band information and IRI data. These models can be used for other case studies on a nationwide scale; however, it must be noted that the IRI measurements were gathered under clear weather conditions between $10^{\circ} \mathrm{C}$ and $30^{\circ} \mathrm{C}$ with a long chassis car (CHANGAN-CS35). The altitude of the study area is approximately $1500 \mathrm{~m}$ above mean sea level (MSL), and the average speed of the QC was approximately $80 \mathrm{~km} / \mathrm{h}$. Accordingly, the limited transferability of the developed functions to other areas may reduce the road quality mapping capability elsewhere. In other words, for different areas or states, the development of domestic functions compatible with geographical specifications and road construction regulations is highly recommended.

Author Contributions: Conceptualization, S.K. and M.M.; methodology, S.K. and M.M.; software, S.K.; validation, S.K.; formal analysis, S.K.; investigation, S.K. and M.M.; resources, S.K.; data curation, S.K.; writing — original draft preparation, S.K.; visualization, S.K.; supervision, S.K. and M.M.; writing—review and editing, M.M.; project administration, S.K. and M.M.; funding acquisition M.M. All authors have read and agreed to the published version of the manuscript.

Funding: This work was supported by University of Tabriz, International and Academic Cooperation Directorate, in the framework of TabrizU-300 program, and also Japanese Society for the Promotion of Science (JSPS) Grants-in-Aid for Scientific Research (KAKENHI), grant number 20H02411 and $18 \mathrm{H} 00769$.

Institutional Review Board Statement: Not applicable.

Informed Consent Statement: Not applicable.

Acknowledgments: The Sentinel-2 images were provided by the European Space Agency (ESA).

Conflicts of Interest: The authors declare no conflict of interest. 


\section{References}

1. $\quad$ Burningham, S.; Stankevich, N. Why Road Maintenance Is Important and How to Get It Done; Transport Notes Series; No. TRN 4; License: CC BY 3.0 IGO; World Bank: Washington, DC, USA, 2005; Available online: https:/ / openknowledge.worldbank.org/ handle/10986/11779 (accessed on 20 October 2020).

2. Hicks, R.; Mahoney, J. Collection and use of pavement condition data. NCHRP Synth. 1981, 76, 1-25.

3. Meyer, F.J.; Ajadi, O.A.; Hoppe, E.J. Studying the Applicability of X-Band SAR Data to the Network-Scale Mapping of Pavement Roughness on US Roads. Remote Sens. 2020, 12, 1507. [CrossRef]

4. Karimzadeh, S.; Matsuoka, M. Remote Sensing X-Band SAR Data for Land Subsidence and Pavement Monitoring. Sensors 2020, 20, 4751. [CrossRef] [PubMed]

5. Sayers, M.W. On the Calculation of International Roughness Index from Longitudinal Road Profile; Transportation Research Board: Washington, DC, USA, 1995; pp. 1-12.

6. Karimzadeh, S.; Matsuoka, M. A Weighted Overlay Method for Liquefaction-Related Urban Damage Detection: A Case Study of the 6 September 2018 Hokkaido Eastern Iburi Earthquake, Japan. Geosciences 2018, 8, 487. [CrossRef]

7. Karimzadeh, S.; Matsuoka, M. Ground Displacement in East Azerbaijan Province, Iran, Revealed by L-band and C-band InSAR Analyses. Sensors 2020, 20, 6913. [CrossRef]

8. Milillo, P.; Bürgmann, R.; Lundgren, P.; Salzer, J.; Perissin, D.; Fielding, E.; Biondi, F.; Milillo, G. Space geodetic monitoring of engineered structures: The ongoing destabilization of the Mosul dam, Iraq. Sci. Rep. 2016, 6, 37408. [CrossRef]

9. Karimzadeh, S.; Matsuoka, M.; Ogushi, F. Spatiotemporal deformation patterns of the Lake Urmia Causeway as characterized by multisensor InSAR analysis. Sci. Rep. 2018, 8, 5357. [CrossRef]

10. Schnebele, E.; Tanyu, B.F.; Cervone, G.; Waters, N. Review of remote sensing methodologies for pavement management and assessment. Eur. Transp. Res. Rev. 2015, 7, 7. [CrossRef]

11. Emery, W.; Singh, M.C. Large-Area Road-Surface Quality and Land-Cover Classification Using Very-High Spatial Resolution Aerial and Satellite Data; RITARS-12-H-CUB; U.S. Department of Transportation: San Francisco, CA, USA, 2013.

12. Emery, W.; Yerasi, A.; Longbotham, N.; Pacifici, F. Assessing paved road surface condition with high resolution satellite imagery. In Proceedings of the IEEE International Geoscience and Remote Sensing Symposium (IGARSS), Quebec, QC, Canada, 13-18 July 2014; pp. 1-4.

13. Suanpaga, W.; Yoshikazu, K. Riding quality model for asphalt pavement monitoring using phase array type L-band synthetic aperture radar (PALSAR). Remote Sens. 2010, 2, 2531-2546. [CrossRef]

14. Mohammadi, A.; Karimzadeh, S.; Valizadeh Kamran, K.; Matsuoka, M. Extraction of Land Information, Future Landscape Changes and Seismic Hazard Assessment: A Case Study of Tabriz, Iran. Sensors 2020, 20, 7010. [CrossRef]

15. Nhu, V.-H.; Mohammadi, A.; Shahabi, H.; Ahmad, B.B.; Al-Ansari, N.; Shirzadi, A.; Geertsema, M.; Kress, V.R.; Karimzadeh, S.; Valizadeh Kamran, K.; et al. Landslide Detection and Susceptibility Modeling on Cameron Highlands (Malaysia): A Comparison between Random Forest, Logistic Regression and Logistic Model Tree Algorithms. Forests 2020, 11, 830. [CrossRef]

16. Yagi, K. Type Roughness Measurement and Cracking Detection Method by Using Smartphone. In Proceedings of the World Conference on Pavement and Asset Management, Milan, Italy, 12-16 June 2017.

17. Sayers, M.; Gillespie, T.D. The Ann Arbor Road Profilometer Meeting; U.S. Department of Transportation: Washington, DC, USA, 1986; p. 226.

18. Liu, J.; Qin, Q.; Li, J.; Li, Y. Rural Road Extraction from High-Resolution Remote Sensing Images Based on Geometric Feature Inference. ISPRS Int. J. Geo-Inf. 2017, 6, 314. [CrossRef]

19. Alshaikhli, T.; Liu, W.; Maruyama, Y. Automated Method of Road Extraction from Aerial Images Using a Deep Convolutional Neural Network. Appl. Sci. 2019, 9, 4825. [CrossRef]

20. Mattyus, G.; Wang, S.; Fidler, S.; Urtasun, R. Enhancing Road Maps by Parsing Aerial Images Around the World. In Proceedings of the 2015 IEEE International Conference on Computer Vision (ICCV), Santiago, Chile, 7-13 December 2015; IEEE Computer Society: Columbia, DC, USA, 2015; pp. 1689-1697.

21. Luque, B.; Morros, J.R.; Ruiz-Hidalgo, J. Spatio-temporal Road Detection from Aerial Imagery using CNNs. In Proceedings of the 12th International Joint Conference on Computer Vision, Imaging and Computer Graphics Theory and Applications, Port, Portugal, 27 February-1 March 2017; SCITEPRESS—Science and Technology Publications: Porto, Portugal, 2017 ; pp. 493-500.

22. Cira, C.-I.; Alcarria, R.; Manso-Callejo, M.-Á.; Serradilla, F. A Deep Learning-Based Solution for Large-Scale Extraction of the Secondary Road Network from High-Resolution Aerial Orthoimagery. Appl. Sci. 2020, 10, 7272. [CrossRef]

23. Kienast-Brown, L.; Boettinger, J.L. Applying the Optimum Index Factor to Multiple Data Types in Soil Survey. In Digital Soil Mapping; Springer: Dordrecht, The Netherlands, 2010; pp. 385-398.

24. Chavez, P.S.; Berlin, G.L.; Sowers, B. Statistical method for selecting Landsat MSS ratios. J. Appl. Photogr. Eng. 1982, 8, 23-30.

25. Chavez, P.S.; Guptill, S.C.; Bowell, J.A. Image processing techniques for thematic mapper data. Proc. ASPRS-ACSM Tech. Pap. 1984, 2, 728-742.

26. Karimzadeh, S.; Mastuoka, M. Building Damage Assessment Using Multisensor Dual-Polarized Synthetic Aperture Radar Data for the 2016 M 6.2 Amatrice Earthquake, Italy. Remote Sens. 2017, 9, 330. [CrossRef]

27. Matsuoka, M.; Nojima, N. Building Damage Estimation by Integration of Seismic Intensity Information and Satellite L-band SAR Imagery. Remote Sens. 2010, 9, 2111-2126. [CrossRef] 
28. Huang, H.; Li, Z.; Pan, Y. Multi-Feature Manifold Discriminant Analysis for Hyperspectral Image Classification. Remote Sens. 2019, 11, 651. [CrossRef]

29. Karimzadeh, S.; Matsuoka, M.; Kuang, J.; Ge, L. Spatial Prediction of Aftershocks Triggered by a Major Earthquake: A Binary Machine Learning Perspective. ISPRS Int. J. Geo-Inf. 2019, 8, 462. [CrossRef]

30. Arai, K. Bi-Directional Reflectance Distribution Function: BRDF Effect on Un-mixing, Category Decomposition of the Mixed Pixel (MIXEL) of Remote Sensing Satellite Imagery Data. Int. J. Adv. Res. Artif. Intell. (IJARAI) 2013, 2, 19-23. [CrossRef]

31. Arai, K. Inversion techniques for proportion estimation of mixels in high resolution satellite image analysis. Adv. Space Res. 1994, 14, 177-186. [CrossRef]

32. Sun, L. Simulation of pavement roughness and IRI based on power spectral density. Math. Comput. Simul. 2003, 61, 77-88. [CrossRef] 